\title{
Tentative Colistin Epidemiological Cut-Off Value for Salmonella spp.
}

Agersø, Yvonne; Torpdahl, Mia; Zachariasen, Camilla; Seyfarth, Anne Mette; Hammerum, Anette M.; Nielsen, Eva Møller

\author{
Published in: \\ Foodborne Pathogens and Disease
}

Link to article, DOI:

10.1089/fpd.2011.1015

Publication date:

2012

Document Version

Publisher's PDF, also known as Version of record

Link back to DTU Orbit

Citation (APA):

Agersø, Y., Torpdahl, M., Zachariasen, C., Seyfarth, A. M., Hammerum, A. M., \& Nielsen, E. M. (2012).

Tentative Colistin Epidemiological Cut-Off Value for Salmonella spp. Foodborne Pathogens and Disease, 9(4), 367-369. https://doi.org/10.1089/fpd.2011.1015

\section{General rights}

Copyright and moral rights for the publications made accessible in the public portal are retained by the authors and/or other copyright owners and it is a condition of accessing publications that users recognise and abide by the legal requirements associated with these rights.

- Users may download and print one copy of any publication from the public portal for the purpose of private study or research.

- You may not further distribute the material or use it for any profit-making activity or commercial gain

- You may freely distribute the URL identifying the publication in the public portal 


\title{
Tentative Colistin Epidemiological Cut-Off Value for Salmonella spp.
}

\author{
Yvonne Agers $\emptyset^{1}$, Mia Torpdahl, ${ }^{2}$ Camilla Zachariasen, ${ }^{2}$ AnneMette Seyfarth, \\ Anette M. Hammerum, ${ }^{2}$ and Eva Møller Nielsen ${ }^{2}$
}

\begin{abstract}
The objective of this research was to determine minimal inhibitory concentration (MIC) population distributions for colistin for Salmonella on subtype level. Furthermore, we wanted to determine if differences in MIC for colistin could be explained by mutations in $p m r A$ or $p m r B$ encoding proteins involved in processes that influence the binding of colistin to the cell membrane. During 2008-2011, 6,583 Salmonella enterica subsp. enterica isolates of human origin and 1931 isolates of animal/meat origin were collected. The isolates were serotyped, and susceptibility was tested towards colistin (range 1-16 mg/L). Moreover, 37 isolates were tested for mutations in pmrA and $p m r B$ by polymerase chain reaction (PCR) and DNA sequencing. MIC distribution for colistin at serotype level showed that Salmonella Dublin $(n=198)$ followed by Salmonella Enteritidis $(n=1247)$ were less susceptible than "other" Salmonella serotypes originating from humans $(n=5,274)$ and Salmonella Typhimurium of animal/meat origin $(n=1794)$. MIC was $\leq 1 \mathrm{mg} / \mathrm{L}$ for $98.9 \%$ of "other" Salmonella serotypes originating from humans, $99.4 \%$ of Salmonella Typhimurium, $61.3 \%$ of Salmonella Enteritidis, and $12.1 \%$ of Salmonella Dublin isolates. Interestingly, Salmonella Dublin and Salmonella Enteritidis belong to the same O-group (O:1, 9,12), suggesting that surface lipopolysaccharides (LPS) of the cell (O-antigen) play a role in colistin susceptibility. The epidemiological cut-off value of $>2 \mathrm{mg} / \mathrm{L}$ for colistin suggested by European Committee on Antimicrobial Susceptibility Testing (EUCAST) is placed inside the distribution for both Salmonella Dublin and Salmonella Enteritidis. All tested Salmonella Dublin isolates, regardless of MIC colistin value, had identical $p m r A$ and $p m r B$ sequences. Missense mutations were found only in pmrA in one Salmonella Reading and in pmrB in one Salmonella Concord isolate, both with MIC of $\leq 1$ for colistin. In conclusion, our study indicates that missense mutations are not necessarily involved in increased MICs for colistin. Increased MICs for colistin seemed to be linked to specific serotypes (Salmonella Dublin and Salmonella Enteritidis). We recommend that Salmonella with MIC of $>2 \mathrm{mg} / \mathrm{L}$ for colistin be evaluated on the serovar level.
\end{abstract}

\section{Introduction}

$\mathbf{T}$

HE INTERPRETIVE CRITERIA are crucial when determining bacteria as either resistant or sensitive to an antimicrobial drug. The European Committee on Antimicrobial Susceptibility Testing (EUCAST, 2011) presents a clinical breakpoint and an epidemiological cut-off value of $>2 \mathrm{mg} / \mathrm{L}$ for colistin, based on a few isolates, whereas Salmonella isolates with a Minimal Inhibitory Concentration (MIC) of $\leq 2 \mathrm{mg} / \mathrm{L}$ should be reported as sensitive or wildtype. Clinical Laboratory Standards Institute (CLSI) defines clinical breakpoints including also treatment efficacy (CLSI, 2011). The epidemiological cut-off value is defined based on MIC population distributions of Salmonella spp. (EUCAST, 2011).
Previously, colistin was used less frequently due to toxicity, but recently it has become the first choice treatment of severe infections involving some multidrug resistant Gram-negative bacteria (Lim et al., 2010).

We hypothesized that colistin MIC population distributions of Salmonella on serotype level differ and MICs of colistin are increased in Salmonella isolates with missense mutations in pmrA or $p m r B$ encoding products involved in processes that influence cell membrane binding of colistin (Roland et al., 1993; Sun et al., 2009).

The objective of this work was to determine MIC population distributions for Salmonella on subtype level and to determine if differences in MIC could be explained by mutations in $p m r A$ or $p m r B$.

\footnotetext{
${ }^{1}$ National Food Institute, Technical University of Denmark, Lyngby, Denmark.

${ }^{2}$ Statens Serum Institut, Copenhagen, Denmark.
} 


\section{Methods}

From 2008 to 2011, the MIC for colistin (concentration range $1-16 \mathrm{mg} / \mathrm{L}$ ) was determined using commercially dehydrated antimicrobial agents in microtiter wells (Sensititre ${ }^{\text {TM}}$; TREK Diagnostic Systems Ltd., West Sussex, United Kingdom) as part of the Danish Integrated Antimicrobial Resistance Monitoring and Research Programme (DANMAP) (CLSI, 2008; DANMAP, 2008-2010). From human patients in Denmark, 6,583 Salmonella enterica subsp. enterica isolates were tested. Among these isolates, the most common serotypes were Salmonella Typhimurium (3,281 isolates, including monophasic variants S. 4,5,12:i:- and S. 4,12:i:-), Salmonella Enteritidis (1,178 isolates), and Salmonella Dublin (131 isolates). The remaining 1,993 isolates belonged to 198 different serotypes. Furthermore, 1,795 Salmonella Typhimurium, 69 Salmonella Enteritidis, and 67 Salmonella Dublin from food animals and raw meat were tested.

In order to determine if differences in MIC were caused by mutations in $p m r A$ and $p m r B$, we looked for mutations in 37 isolates (with MIC and identification verified) of serovar Salmonella Dublin $(n=22)$, Salmonella Enteritidis $(n=14)$, and Salmonella Typhimurium $(n=1)$ from human infections in 2009 with MIC values of 2, 4, or $8 \mathrm{mg} / \mathrm{L}$. In addition, one isolate of each of the above-mentioned three serotypes, plus one Salmonella Concord and one Salmonella Reading with MIC of $\leq 1$ were included to look for differences in susceptible isolates of five different serotypes. The $p m r A$ and $p m r B$ genes were sequenced using the primers reported by Sun et al. (2009) and additional new primers designed to fully include $\mathrm{pmr} B$ (forward: 5'-CGACGGACTCAATCTCAAG-3'; reverse: 5'GCATATACATAATTTGCGCGA-3').

\section{Results and Discussion}

The MIC distribution for all Salmonella isolates is shown in Figure 1A; the MIC distributions for Salmonella Dublin, Salmonella Enteritidis, Salmonella Typhimurium (food animals and raw meats), and "other" serotypes originating from humans are presented in Figure 1B. Salmonella Dublin followed by Salmonella Enteritidis were less susceptible than the other Salmonella serotypes. Interestingly, Salmonella Dublin and Salmonella Enteritidis have the same O-antigen formula (O:1, 9,12).

Therefore, we also looked at the MIC distribution of colistin for available Salmonella Napoli $(n=18)$ and Salmonella Panama $(n=30)$, which also have this O-antigen formula. Although these two serovars also seemed less susceptible to colistin than Salmonella in general, the trend was not as pronounced; MIC was $\leq 1 \mathrm{mg} / \mathrm{L}$ for $98.9 \%$ of "other" Salmonella serotypes of human origin, $99.4 \%$ for Salmonella Typhimurium from animal/meat origin, $89 \%$ for Salmonella Napoli, $80 \%$ for Salmonella Panama, $61.3 \%$ for Salmonella Enteritidis, and $12.1 \%$ for Salmonella Dublin. This suggests that the surface lipopolysaccharides (LPS) of the cell (O-antigen) play a role in colistin susceptibility. The epidemiological cut-off value suggested by EUCAST ( $>2 \mathrm{mg} / \mathrm{L}$ ) is placed inside the distribution for both Salmonella Dublin and Salmonella Enteritidis. Due to the low number of especially Salmonella Dublin when compared to other Salmonella, these differences related to serotypes are overlooked when merging all Salmonella into the same distribution (Fig. 1).

Colistin targets the cell envelope, and the interaction between the cationic polypeptide and negatively charged LPS
A
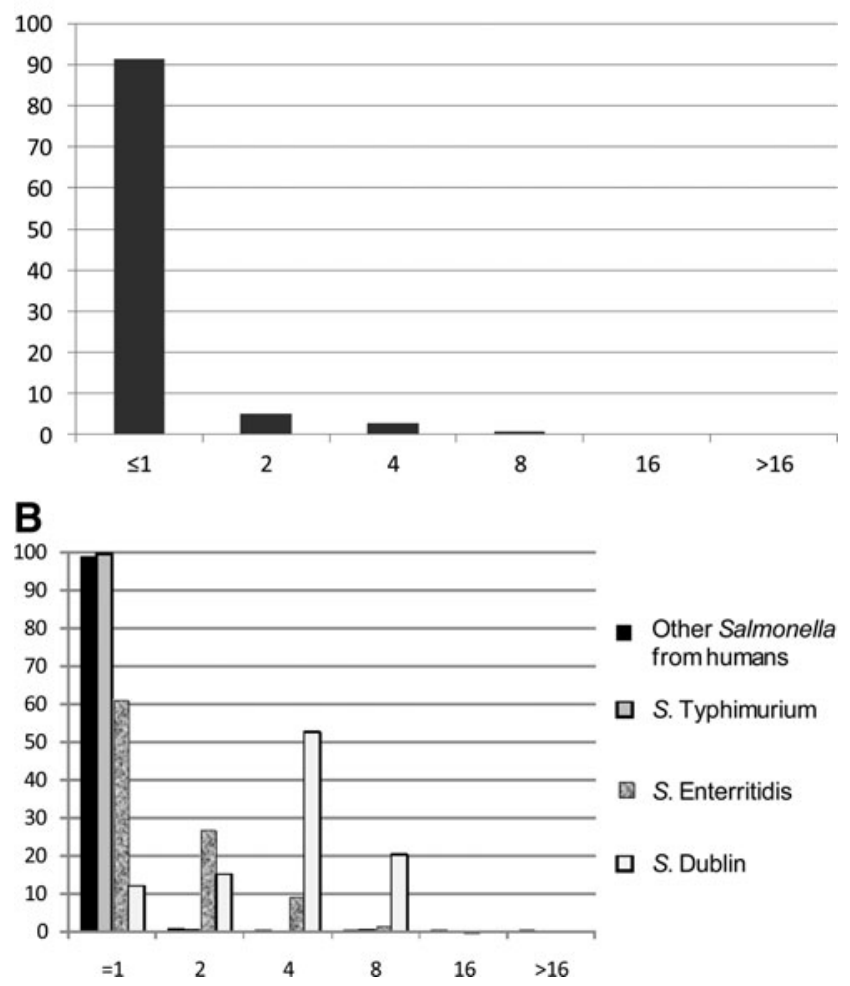

FIG. 1. (A) Percentage distribution of minimal inhibitory concentration (MIC) of colistin determined for all Salmonella serovars analyzed $(n=8,514)$. (B) MIC distribution of "other" Salmonella serovars from humans $(n=5,274)$, Salmonella Typhimurium from food animals and raw meats $(n=1,795)$, Salmonella Enteritidis $(n=1,247)$, and Salmonella Dublin $(n=198)$.

leads to a disturbance of the outer membrane and a subsequent increase in the permeability of the cell envelope resulting in death (Schindler and Osborn, 1979; Sun et al., 2009). Increase in MIC for Salmonella Typhimurium has been mapped to $p m r A$ and $p m r B$, which constitute a two-component regulatory system. PmrB is a sensor histidine kinase, and PmrA is the cognate response regulator. The modifications in these genes influence the regulation of proteins, which makes the LPS less negatively charged and thereby decrease the binding of colistin (Roland et al., 1993; Sun et al., 2009).

Sun et al. (2009) found 27 missense mutations in $p m r B$ and pmrA that lead to an increased MIC to colistin of up to $4.4 \mathrm{mg} /$ L. For the isolates tested in the present study, all Salmonella Dublin isolates, regardless of the MIC value, had identical pmrA and $p m r B$ sequences. Compared to the Salmonella LT2 strain (GenBank accession no. NC003197), the Salmonella Dublin isolates had a point mutation in $p m r B$ position $25(\mathrm{~A} \rightarrow$ C), leading to an amino acid change in position 9 from threonine to proline. None of the other isolates had this mutation. In addition, five and six synonymous point mutations were seen in pmrB Salmonella Dublin and Salmonella Enteritidis isolates, respectively (two of them were identical, positions 492 and 750). No missense mutation was present in $p m r A$, but synonymous point mutations were seen at positions 177 and 322 in Salmonella Dublin $(\mathrm{C} \rightarrow \mathrm{T})$ and at positions 322 and 414 in Salmonella Enteritidis $(\mathrm{C} \rightarrow \mathrm{T})$. In addition, missense 
mutations in pmrA were found in one Salmonella Reading isolate, and a missense mutation in $p m r B$ was found in one Salmonella Concord isolate; both had MIC of $\leq 1$. Therefore, missense mutations are not necessarily involved in increased MICs for colistin.

In conclusion, since increased MIC seemed linked to serotype, we recommend that isolates with MIC of $>2 \mathrm{mg} / \mathrm{L}$ for colistin in Salmonella spp. be evaluated on the serotype level. More detailed data when making population distributions (such as interpreting results based on the subtype level and looking for resistance mechanisms) may be necessary when determining interpretive criteria.

\section{Acknowledgments}

The technical staff at Statens Serum Institut and the National Food Institute, Technical University of Denmark are thanked for their assistance. We would like to thank Gunnar Kahlmeter for useful discussion. Data will be submitted to EUCAST for use in population distributions for Salmonella on serovar level. This work was supported in part by the Danish Ministry of Science, Technology and Innovation and the Danish Ministry of Health and Prevention as part of the Danish Integrated Antimicrobial Resistance Monitoring and Research Programme (DANMAP).

\section{Disclosure Statement}

No competing financial interests exist.

\section{References}

[CLSI] Clinical Laboratory Standards Institute. Performance Standards for Antimicrobial Susceptibility Testing. Eighteenth Informational Supplement. Document M100-S18. Wayne, PA: CLSI, 2008.

[CLSI] Clinical Laboratory Standards Institute. Performance Standards for Antimicrobial Susceptibility Testing. Twenty-First Informational Supplement. Document M100-S21. Wayne, PA: CLSI, 2011.

[EUCAST] European Committee on Antimicrobial Susceptibility Testing. EUCAST clinical MIC breakpoints. 2011. Available at: http://www.eucast.org/clinical_breakpoints, accessed March 31, 2011.

[DANMAP] Danish Integrated Antimicrobial Resistance Monitoring and Research Programme. DANMAP 2008-Use of antimicrobial agents and occurrence of antimicrobial resistance in bacteria from food animals, foods and humans in Denmark. 1999. Available at: http://www.danmap.org, accessed March 31, 2011.

[DANMAP] Danish Integrated Antimicrobial Resistance Monitoring and Research Programme. DANMAP 2009-Use of antimicrobial agents and occurrence of antimicrobial resistance in bacteria from food animals, foods and humans in Denmark. 2000. Available at: http://www.danmap.org, accessed March 31, 2011.

[DANMAP] Danish Integrated Antimicrobial Resistance Monitoring and Research Programme. DANMAP 2010-Use of antimicrobial agents and occurrence of antimicrobial resistance in bacteria from food animals, foods and humans in Denmark. 2001. Available at: http://www.danmap.org, accessed September 30, 2011.

Lim LM, Ly N, Anderson D, et al. Resurgence of colistin: A review of resistance, toxicity, pharmacodynamics, and dosing. Pharmacotherapy 2010;30:1279-1291.

Roland KL, Martin LE, Esther CR, et al. Spontaneous pmrA mutants of Salmonella Typhimurium LT2 define a new twocomponent regulatory system with a possible role in virulence. J Bacteriol 1993;175:4154-4164.

Schindler M, Osborn MJ. Interaction of divalent cations and polymyxin B with lipopolysaccharide. Biochemistry 1979;18: 4425-4164.

Sun S, Negrea A, Rhen M, et al. Genetic analysis of colistin resistance in Salmonella enterica serovar Typhimurium. Antimicrob Agents Chemother 2009;53:2298-2305.

Address correspondence to: Yvonne Agersø, Ph.D. National Food Institute Technical University of Denmark Kemitorvet Building 204 DK-2800 Lyngby, Denmark

E-mail: yvoa@food.dtu.dk 